\title{
Una demostración del volumen y la medida superficial de la bola unidad
}

\author{
Marlon Josué ReCARTE \\ Universidad Nacional Autónoma de Honduras en el Valle de Sula, mail: recarte27@hotmail.com
}

Recibido: 06 de Febrero de 2016 / Aceptado: 01 de Mayo de 2016

\section{Resumen}

\begin{abstract}
In statistical physics it is necesary express some results using the volume of a hypersphere, which is a generalization of a sphere for a dimention larger than three. On the other hand, different models of the shape of the universe has been proposed, particularly some of then consider a curved shape and can be modeled as a hypersphere. In this paper we focus on obtaining the volume and surface measurement of a hypersphere of unit radius often called unit ball.
\end{abstract}

Keywords: Hypersphere, unit ball

En física estadística a menudo se tiene la necesidad de expresar algunos resultados utilizando el volumen de una hiperesfera, que es una generalización de una esfera para dimensiones mayores a tres. Por otro lado, existen diversos modelos sobre la forma del universo, particularmente algunos consideran una forma curva y dicha forma puede ser modelada como una hiperesfera. En este trabajo nos concentramos en obtener el volumen y la medida superficial de una hiperesfera de radio unitario llamada muchas veces bola unidad.

Palabras claves: Hiperesfera, bola unidad

\section{ESFERA Y BOLA UNIDAD}

$\mathrm{D}$ ENOTEMos $B^{d}$ la bola unitaria en el espacio euclídeo $\mathbb{R}^{d}$, definida como:

$$
B^{d}=\left\{x=\left(x_{1}, x_{2} \ldots, x_{d}\right) \in \mathbb{R}^{d}:\|x\| \leq 1\right\}
$$

donde $\|\cdot\|$ denota la norma usual euclídea

$$
\|x\|=\sqrt{x_{1}^{2}+\cdots+x_{d}^{2}}
$$

Denotamos $S^{d-1}$ la esfera unidad,

$$
S^{d-1}=\left\{x \in \mathbb{R}^{d}:\|x\|=1\right\}
$$

Al considerar $d=1$ la bola unidad es un intervalo, para $d=2$ se tiene un círculo y para $d=3$ una esfera, ver figura 1

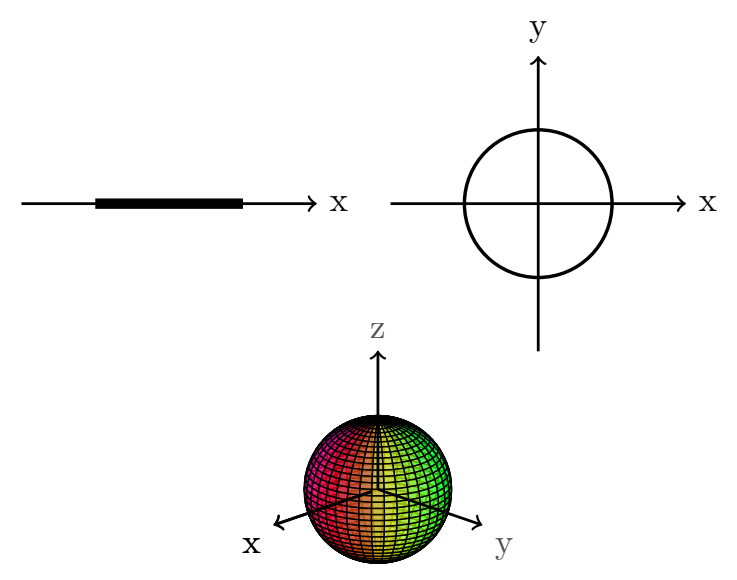

Figura 1: Bolas unitarias para $d=1,2,3$.

A. Área superficial y volumen de la bola unidad

En la literatura existen diversas pruebas para el cálculo del volumen y la medida superficial de la bola unidad (llamada algunas veces la $\mathbf{d}$-esfera). El artículo clásico de Blumenson [3] presenta las coordenadas esféricas ndimensionales o coordenadas polares generalizadas (que presentaremos luego) incluyendo además una deducción de la medida superficial y el volumen a partir de un cambio de variable en dichas coordenadas. Muller 5 presenta una prueba del mismo resultado a partir de una relación de recurrencia. Smith y Vamanamurthy [6] aborda ese problema utilizando múltiples técnicas de cálculo. La demostración que presento en este artículo utiliza el trun- 
camiento de un elemento del espacio euclideo, este tipo de cambio de variable (junto con las propiedades de las funciones Beta y Gamma) es útil en la teoría de polinomios ortogonales multivariables para probar relaciones de ortogonalidad (ver 4]) y [2]).

Consideremos la integral sobre la bola unidad, dicha integral representa el volumen de la $\mathbf{d}$-esfera:

$$
V_{d}=\int_{B^{d}} d V=\int_{B^{d}} \prod_{j=1}^{d} d x_{j}
$$

Asociado con $x=\left(x_{1}, x_{2}, \cdots, x_{d}\right) \in \mathbb{R}^{d}$ para cada $j$, definimos $x_{(j)}$, el truncamiento de $x$, esto es: $x_{(0)}=0$,

$$
x_{(j)}=\left(x_{1}, x_{2}, \cdots, x_{j}\right) \text { para } 1 \leq j \leq d
$$

Nótese que $x_{(d)}=x$.

Necesitamos hacer uso de la siguiente fórmula:

$$
\int_{B^{d}} f(x) d x=\int_{B^{d}} f\left(x_{(d-1)}, x_{d}\right) d x
$$

Sea $y_{i} \in \mathbb{R}$ tales que se cumple

$$
x_{i}=y_{i} \sqrt{1-\left\|x_{(i-1)}\right\|^{2}}
$$

entonces se verifica que:

$$
\begin{aligned}
& \left(1-\left\|x_{(0)}\right\|^{2}\right)=1 \\
& \left(1-\left\|x_{(1)}\right\|^{2}\right)=\left(1-y_{1}^{2}\right)\left(1-y_{2}^{2}\right) \\
& \left(1-\left\|x_{(3)}\right\|^{2}\right)=\left(1-y_{1}^{2}\right)\left(1-y_{2}^{2}\right)\left(1-y_{3}^{2}\right)
\end{aligned}
$$

Aseveramos que

$$
\left(1-\left\|x_{(d)}\right\|^{2}\right)=\prod_{i=1}^{d}\left(1-y_{i}^{2}\right)
$$

Supongamos que es válido para $i=d-1$

$$
\begin{aligned}
\left(1-\left\|x_{(d-1)}\right\|^{2}\right) & =\prod_{i=1}^{d-1}\left(1-y_{i}^{2}\right) \\
& =\left[\left(1-y_{d-1}^{2}\right)\left(1-\left\|x_{(d-2)}\right\|^{2}\right)\right],
\end{aligned}
$$

ahora probémoslo para $i=d$ :

$$
\begin{aligned}
\left(1-\left\|x_{(d)}\right\|^{2}\right) & =\left(1-y_{d}^{2}\right)\left(1-\left\|x_{(d-1)}\right\|^{2}\right) \\
& =\left(1-y_{d}^{2}\right) \prod_{i=1}^{d-1}\left(1-y_{i}^{2}\right) \\
& =\prod_{i=1}^{d}\left(1-y_{i}^{2}\right)
\end{aligned}
$$

Notemos que:

$$
\prod_{j=1}^{d}\left(1-\left\|x_{(j-1)}\right\|^{2}\right)^{\frac{1}{2}}=\prod_{j=1}^{d}\left(1-y_{j}^{2}\right)^{\frac{d-j}{2}} .
$$

Retomando la integral inicial, tendremos:

$$
\begin{aligned}
V_{d} & =\int_{B^{d}} \prod_{j=1}^{d} d x_{j} \\
& =\int_{-1}^{1} \prod_{j=1}^{d}\left(1-y_{j}^{2}\right)^{\frac{d-j}{2}} d y_{j} \\
& =\prod_{j=1}^{d} \int_{-1}^{1}\left(1-y_{j}^{2}\right)^{\frac{d-j}{2}} d y_{j} .
\end{aligned}
$$

Al realizar el cambio de variable, $2 t=1-y_{j}$

$$
\begin{aligned}
V_{d} & =\prod_{j=1}^{d} \int_{-1}^{1}\left(1-y_{j}^{2}\right)^{\frac{d-j}{2}} d y_{j} \\
& =\prod_{j=1}^{d} \int_{0}^{1} 2^{d-j+1} t^{\frac{d-j}{2}}(1-t)^{\frac{d-j}{2}} d t \\
& =\prod_{j=1}^{d} 2^{d-j+1} \prod_{j=1}^{d} \int_{0}^{1} t^{\frac{d-j}{2}}(1-t)^{\frac{d-j}{2}} d t .
\end{aligned}
$$

La expresión anterior queda en términos la función Beta $\mathcal{B}(a, b)$ que está definida por:

$$
\mathcal{B}(a, b)=\int_{0}^{1} t^{a-1}(1-t)^{b-1} d t
$$

obteniéndose

$$
V_{d}=\prod_{j=1}^{d} 2^{d-j+1} \prod_{j=1}^{d} \mathcal{B}\left(\frac{d-j}{2}+1, \frac{d-j}{2}+1\right)
$$

Calcularemos cada producto por separado:

$$
\prod_{j=1}^{d} 2^{d-j+1}=\prod_{k=0}^{d-1} 2^{k+1}
$$

$\prod_{j=1}^{d} \mathcal{B}\left(\frac{d-j}{2}+1, \frac{d-j}{2}+1\right)=\prod_{k=0}^{d-1} \mathcal{B}\left(\frac{k}{2}+1, \frac{k}{2}+1\right)$

Usando la relación entre las funciones beta $\mathcal{B}(a, b)$ y gamma $\Gamma(a)$.

$$
\begin{gathered}
\Gamma(a)=\int_{0}^{\infty} t^{a-1} e^{-t} d t, \\
\mathcal{B}(a, b)=\frac{\Gamma(a) \Gamma(b)}{\Gamma(a+b)},
\end{gathered}
$$

deducimos que

$$
\begin{aligned}
\prod_{k=0}^{d-1} \mathcal{B}\left(\frac{k}{2}+1, \frac{k}{2}+1\right) & =\prod_{k=0}^{d-1} \frac{\Gamma\left(\frac{k}{2}+1\right) \Gamma\left(\frac{k}{2}+1\right)}{\Gamma(k+2)} \\
& =\prod_{k=2}^{d+1} \frac{\Gamma\left(\frac{k}{2}\right) \Gamma\left(\frac{k}{2}\right)}{\Gamma(k)}
\end{aligned}
$$


Al usar la fórmula de duplicación de la función gamma (Ver [1])

$$
\Gamma\left(\frac{k}{2}\right) \Gamma\left(\frac{k}{2}+\frac{1}{2}\right)=2^{1-k} \sqrt{\pi} \Gamma(k),
$$

se tiene

$$
\begin{gathered}
\prod_{k=0}^{d-1} \mathcal{B}\left(\frac{k}{2}+1, \frac{k}{2}+1\right)=\prod_{k=2}^{d+1} \frac{\Gamma\left(\frac{k}{2}\right) \Gamma\left(\frac{k}{2}\right)}{\Gamma(k)} \\
=\prod_{k=2}^{d+1} \frac{2^{1-k} \sqrt{\pi} \Gamma\left(\frac{k}{2}\right)}{\Gamma\left(\frac{k}{2}+\frac{1}{2}\right)} \\
=\prod_{k=2}^{d+1} 2^{1-k} \prod_{k=2}^{d+1} \sqrt{\pi} \prod_{k=2}^{d+1} \frac{\Gamma\left(\frac{k}{2}\right)}{\Gamma\left(\frac{k}{2}+\frac{1}{2}\right)} \\
=\frac{1}{\Gamma\left(\frac{d+2}{2}\right)} \prod_{k=2}^{d+1} 2^{1-k} \prod_{k=2}^{d+1} \sqrt{\pi}
\end{gathered}
$$

Es fácil comprobar que

$$
\prod_{k=2}^{d+1} 2^{1-k} \prod_{k=0}^{d-1} 2^{k+1}=1
$$

Por lo anterior podemos concluir finalmente que

$$
V_{d}=\int_{B^{d}} \prod_{j=1}^{d} d x_{j}=\frac{\pi^{\frac{d}{2}}}{\Gamma\left(\frac{d+2}{2}\right)}
$$

En la figura 2 observamos el gráfico del volumen de la bola unidad para diferentes dimensiones; notemos que el volumen máximo se obtiene para $d=6$.

Al considerar el comportamiento asintótico de la función gamma (ver [1]) se tiene que $V_{d} \rightarrow 0$ cuando $d \longrightarrow \infty$.

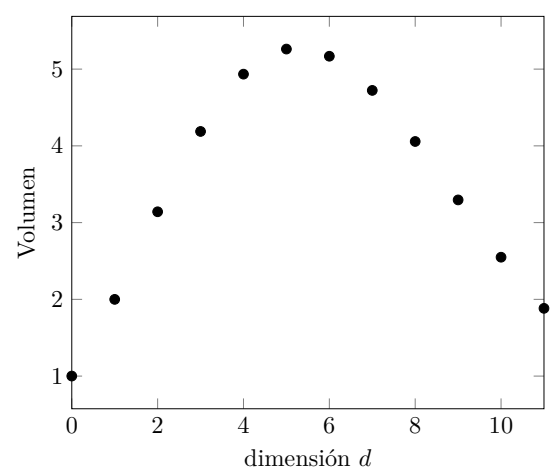

Figura 2: Gráfico del volumén de la bola unidad para diferentes valores de d.
Ahora calcularemos la medida superficial de la bola unidad, $\sigma_{d}$.

$$
\sigma_{d}=2 \int_{B^{d-1}} \sqrt{1+\sum_{i=1}^{d-1}\left(\frac{d x_{n}}{d x_{i}}\right)^{2}} \prod_{j=1}^{d-1} d x_{j}
$$

Para calcular la integral anterior basta con notar que

$$
\begin{aligned}
\sqrt{1+\sum_{i=1}^{d-1}\left(\frac{d x_{n}}{d x_{i}}\right)^{2}} & =\frac{1}{\sqrt{1+\left\|x_{(d-1)}\right\|^{2}}} \\
& =\prod_{i=1}^{d-1}\left(1-y_{i}^{2}\right)^{-1 / 2}
\end{aligned}
$$

debido a esto se tiene

$$
\begin{aligned}
\sigma_{d} & =2 \int_{-1}^{1} \prod_{i=1}^{d-1}\left(1-y_{i}^{2}\right)^{-\frac{1}{2}} \prod_{j=1}^{d-1}\left(1-y_{j}^{2}\right)^{\frac{d-1-j}{2}} d y_{j} \\
& =2 \prod_{j=1}^{d-1} \int_{-1}^{1}\left(1-y_{j}^{2}\right)^{\frac{d-j-2}{2}} d y_{j}
\end{aligned}
$$

La integral anterior es similar a la que se desarrolló en el cálculo del volumen $V_{d}$, entonces si $z=y_{d-1}$

$$
\begin{aligned}
\sigma_{d} & =2 \prod_{j=1}^{d-1} \int_{-1}^{1}\left(1-y_{j}^{2}\right)^{\frac{d-j-2}{2}} d y_{j} \\
& =2 \int_{-1}^{1}\left(1-z^{2}\right)^{-\frac{1}{2}} d z \prod_{j=1}^{d-2} \int_{-1}^{1}\left(1-y_{j}^{2}\right)^{\frac{d-2-j}{2}} d y_{j}
\end{aligned}
$$

Al usar (1) se obtiene

$$
\sigma_{d}=2 \pi \frac{\pi^{\frac{d-2}{2}}}{\Gamma\left(\frac{d}{2}\right)}=\frac{2 \pi^{\frac{d}{2}}}{\Gamma\left(\frac{d}{2}\right)}
$$

El gráfico de la medida superficial se muestra en la figura 3

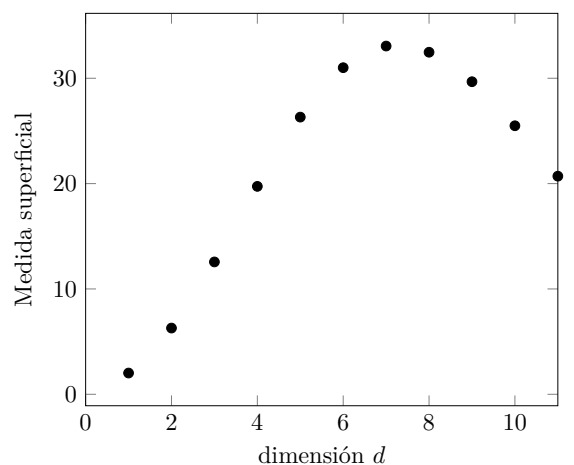

Figura 3: Gráfico de la medida superficial para diferentes valores de $d$. 
B. Coordenadas esféricas generalizadas

Sea $x=r \xi, \quad \xi \in S^{d-1}$. Se definen las coordenadas esféricas generalizadas como una extensión a las coordenadas polares $(d=2)$ y las coordenadas esféricas $(d=3)$ (ver [3])

$$
\begin{aligned}
x_{1} & =r \cos \left(\theta_{d-1}\right), \\
x_{2} & =r \sin \left(\theta_{d-1}\right) \cos \left(\theta_{d-2}\right), \\
& \vdots \\
x_{d-1} & =r \sin \left(\theta_{d-1}\right) \ldots \sin \left(\theta_{2}\right) \cos \left(\theta_{1}\right), \\
x_{d} & =r \sin \left(\theta_{d-1}\right) \ldots \sin \left(\theta_{2}\right) \sin \left(\theta_{1}\right),
\end{aligned}
$$

$\operatorname{con} r>0, \quad 0 \leq \theta_{1} \leq 2 \pi, \quad 0 \leq \theta_{i} \leq \pi$ para $2 \leq i \leq d-2$.

Vale la pena comentar que las definiciones para las coordenadas esféricas generalizadas también se pueden expresar como:

$$
x_{j}=\sqrt{r^{2}-\left\|x_{(j-1)}\right\|^{2}} \cos \left(\theta_{d-j}\right)
$$

para $j=1, \ldots, d-1$.

Puede demostrarse que el Jacobiano de esta transformación es igual a

$$
J=r^{n-1} \prod_{j=1}^{d-2}\left(\sin \theta_{d-j}\right)^{d-j-1} .
$$

Nótese que

$$
V_{d}=\int_{B^{d}} r^{n-1} d r d \omega
$$

donde

$$
d \omega=\prod_{j=1}^{d-2}\left(\sin \theta_{d-j}\right)^{d-j-1} d \theta_{1} d \theta_{2} \cdots d \theta_{d-1} .
$$

además

$$
\sigma_{d}=\int_{S^{d-1}} d \omega
$$

Puede comprobarse que al utilizar las coordenadas esféricas generalizadas se obtiene el volumen de la $d$-esfera de radio $R$

$$
V=\frac{\pi^{\frac{d}{2}}}{\Gamma\left(\frac{d+2}{2}\right)} R^{d}
$$

Al considerar $R=1$ se verifica el resultado obtenido en (1).

\section{REFERENCIAS}

[1] M. ABRAMOWITZ and I. STEGUN. Handbook of Mathematical functions, 9th. ed. Dover Publ, New York, 1970.

[2] K. ATKINSON and W. HAN. Spherical Harmonics and Approximations on the Unit Sphere: An Introduction. Lecture Notes in Mathematics, New York, 2012.

[3] L. E. BLUMENSON. A derivation of $n$-dimentional spherical coordinates. The American Mathematical Monthly. 67, pages 63-66, January 1960.

[4] F. DAI and Y. XU. Approximation Theory and Harmonic Analysis on Spheres and Balls. Springer Monographs in Mathematics, New York, 2013.

[5] C. MULLER. Spherical Harmonics. Springer-Verlag, Berlin, 1966.

[6] D. SMITH and M. VAMANAMURTHY. How small is a unit ball? Mathematics Magazine, 62(2):101-107, 1989. 